\title{
KINETIC MONTE CARLO SIMULATION OF OXYGEN AND CATION DIFFUSION IN YTTRIA-STABILIZED ZIRCONIA
}

Brian Good, Materials and Structures Division, NASA GRC, Cleveland, Ohio

\begin{abstract}
Yttria-stabilized zirconia (YSZ) is of interest to the aerospace community, notably for its application as a thermal barrier coating for turbine engine components. In such an application, diffusion of both oxygen ions and cations is of concern. Oxygen diffusion can lead to deterioration of a coated part, and often necessitates an environmental barrier coating. Cation diffusion in YSZ is much slower than oxygen diffusion. However, such diffusion is a mechanism by which creep takes place, potentially affecting the mechanical integrity and phase stability of the coating. In other applications, the high oxygen diffusivity of YSZ is useful, and makes the material of interest for use as a solidstate electrolyte in fuel cells.

The kinetic Monte Carlo (kMC) method offers a number of advantages compared with the more widely known molecular dynamics simulation method. In particular, $\mathrm{kMC}$ is much more efficient for the study of processes, such as diffusion, that involve infrequent events.

We describe the results of kinetic Monte Carlo computer simulations of oxygen and cation diffusion in YSZ. Using diffusive energy barriers from ab initio calculations and from the literature, we present results on the temperature dependence of oxygen and cation diffusivity, and on the dependence of the diffusivities on yttria concentration and oxygen sublattice vacancy concentration. We also present results of the effect on diffusivity of oxygen vacancies in the vicinity of the barrier cations that determine the oxygen diffusion energy barriers.
\end{abstract}




\section{Kinetic Monte Carlo Simulation of Oxygen and Cation Diffusion in Yttria- Stabilized Zirconia.}

Brian Good, Structures and Materials Division NASA GRC, Cleveland, Ohio. 


\section{Motivation}

Yttria-stabilized zirconia (YSZ) is of interest to the aerospace community, notably for its application as a thermal barrier coating for turbine engine components. In such an application, diffusion of both oxygen ions and cations is of concern.Oxygen diffusion can lead to deterioration of a coated part, and often necessitates an additional antioxidation coating. On the other hand, the high oxygen diffusivity of YSZ makes the material of interest for use as a solid-state electrolyte in fuel cells. Cation diffusion in YSZ is much slower than oxygen diffusion. However, such diffusion is a mechanism by which creep takes place, potentially affecting mechanical integrity and phase stability. 


\section{Why Kinetic Monte Carlo?}

Currently, molecular dynamics (MD) is the method of choice when examining the dynamical behavior of many-atom systems.MD produces detailed trajectories of atoms, but is typically limited to time scales on the order of nanoseconds. When the phenomenon under study involves so-called "infrequent events," MD may be very inefficient.A diffusive hop (the mechanism behind both oxygen ion and cation diffusion) is such an event.KMC is a simulation method designed to deal with infrequent-event systems. It averages out the system's behavior during "uninteresting" times and concentrates on the events of interest, and is typically much faster than MD for such systems.It requires a knowledge of all the "interesting" events accessible to the system. It does not provide detailed atomic trajectories. 
We describe the results of kinetic Monte Carlo computer simulations of oxygen and cation diffusion in YSZ. Using diffusive energy barriers from ab initio calculations and from the literature, we present results on the temperature dependence of oxygen and cation diffusivity, and on the dependence of the oxygen diffusivity on $Y$ concentration. We also present preliminary results of the effect on diffusivity of oxygen vacancy clustering near $\mathrm{Y}$ ions. 


\section{kMC, as applied to diffusion}

The sole events of interest are diffusive hops among vacancy sites on the oxygen or cation sublattices.

Because the vibrational frequency is much larger than the average frequency of hops, the system loses its memory of the previous event (here, which of a vacancy's neighbors were involved in the previous hop). Therefore, the events can be considered as independent.

In such cases, the probability per unit time that a vacancy will undergo a diffusive hop is constant, and the probability distribution for the first hop is given by

$$
p(t)=\nu_{\text {tot }} \exp \left(-\nu_{\text {tot }} t\right)
$$

where $\nu_{\text {tot }}$ is the aggregate rate constant for all hops accessible to the vacancy. Because all events are independent, the overall hopping rate is the sum of the individual rates for each accessible hop.

In diffusive hopping, the rates depend on the energy barriers:

$$
\nu_{A B}=\nu^{0} \exp \left(-E_{A B} / k_{B} T\right)
$$

in which $\nu_{A B}$ and $E_{A B}$ are the hopping rate and migration barrier energy for a hop between oxygen or cation sites $A$ and $B$ respectively, and is the frequency factor.

The hopping probability is computed from the hopping rat $E_{A B}=\frac{\nu_{A B}}{\Gamma}$

where $\Gamma$ is the sum of all hopping rates. 
A computational cell is constructed with the appropriate numbers of $Y$ cations and $O$ vacancies distributed randomly, consistent with charge neutrality.

All events accessible to the system are identified and the corresponding event probabilities are computed and collected in an event catalog.

Iterative $\mathrm{kMC}$ procedure:

An event is chosen at random and executed.

The simulation clock is advanced by a time stept $=-\frac{\ln (R)}{\Gamma}$

where $\mathrm{R}$ is a random number greater than zero and less than or equal to unity.

The event probabilities and rates are modified to reflect the new state of the system, and are included in the modified catalog.

When the simulation has run long enough to accumulate statistically useful information, the mean square displacement, averaged over all vacancies, is computed. The vacancy diffusivity is obtained from the mean square hopping distance using the Einstein relation

$$
<R^{2}>=6 D_{\nu} t
$$

and the ionic diffusivity $D_{i}$ is obtained by balancing the number of vacancy and ion hops:

$$
D_{i}=\frac{C_{i v}}{1-C_{i v}} D_{\nu}
$$

where $C_{i v}$ is the concentration of vacancies on the sublattice of the hopping ion. 


\section{Oxygen diffusivity in YSZ}

Oxygen diffusivity of YSZ over the temperature range of interest is typically in the range of $10^{-6}-10^{-7} \mathrm{~cm}^{2} / \mathrm{sec}$.

Diffusivity increases with increasing temperature.

Diffusivity increases with increasing $Y$ concentration at low concentrations, and reaches a maximum at 8-12 mol percent $\mathrm{Y}_{2} \mathrm{O}_{3}$.

Results from experiment

Oishi and Ando find oxygen diffusivities of $0.5-3.0 \times 10^{-6} \mathrm{~cm}^{2} / \mathrm{sec}$ over a temperature range of 1373K-2073K.

Strickler and Carlson find diffusivities in the range of 0.4-4.6 $\times 10^{-6} \mathrm{~cm}^{2} / \mathrm{sec}$, and observe a maximum in the diffusivity as a function of concentration. They find that the maximum occurs at slightly higher concentrations at higher temperatures.

Results from simulation

Khan et al., produce diffusivities of $1.9 \times 10^{-7}$ to $3.0 \times 10^{-6} \mathrm{~cm}^{2} / \mathrm{sec}$ over the temperature range $873 \mathrm{~K}-2073 \mathrm{~K}$.

Krishnamurthy et al., produce diffusivities of $0.1-7.0 \times 10^{-6} \mathrm{~cm}^{2} / \mathrm{sec}$ over a temperature range of $1000-2200 \mathrm{~K}$.

Y.Oishi and K. Ando, in "Transport in Nonstoichiometric Compounds," G. Simkovich and V. Stubican, eds., Plenum Press, New York, 1985, pp189-202.

D. W. Stickler and W. G. Carlson, J. Am. Ceram. Soc. 47, 122 (1964)

M. S. Kahn, M. S. Islam and D. R. Bates, J. Mater. Chem. 8 [10], 2299-2307 1998.

R. Krishnamurthy, Y.-G. Yoon, D. J. Srolovitz and R. Car, J. Am. Ceram. Soc. 87 [10],1821-1830 (2004).

R. Krishnamurthy, D. J. Srolovitz, K. N. Kudin and R. Car, J. Am. Ceram. Soc. 88 [8],2143-2151 (2005). 
Diffusion path is to the nearest neighbor on oxygen sublattice (100 direction). Each site on the oxygen sublattice has four cation neighbors that form a tetragon, and hopping occurs across tetragonal edges, with the hopping oxygen ion passing between two barrier cations.

Barrier energies are assumed to depend only on the two barrier cations. The possible barrier pairs are $\mathrm{Zr}-\mathrm{Zr}$, Zr-Y and $\mathrm{Y}-\mathrm{Y}$.
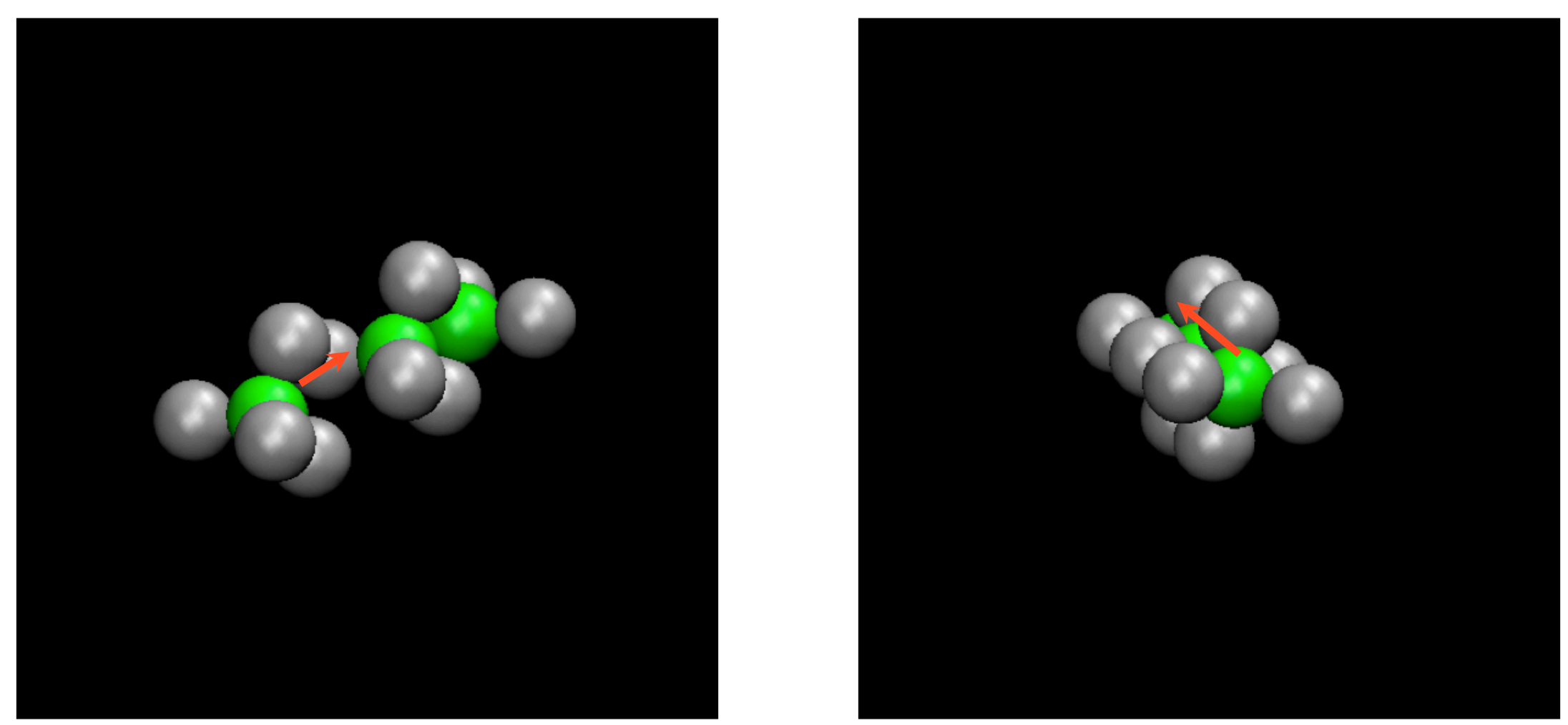

Oxygen ion hopping path. Oxygen ions are green, cations grey.

MD simulations have yielded activation energies of $0.37 \mathrm{eV}$ (Khan) and 0.2-0.8 eV ( $\mathrm{Li}$ and Hafskjold). A tracer diffusion study finds a value of $0.44 \mathrm{eV}$, while results from bulk conductivity and ac impedance spectroscopy give values of 0.79-1.12 eV. 
Energy barriers computed from ab initio Car-Parinello MD by Krishnamurthy et al. are shown below, along with values used in this work, obtained using the Abinit plane wave pseudopotential code with pseudopotentials from the Fritz-Haber Institute. Note that the ordering of the barrier energies is $\mathrm{Zr}-\mathrm{Zr}, \mathrm{Zr}-\mathrm{Y}$ and $\mathrm{Y}-\mathrm{Y}$.

Ab Initio Oxygen Diffusion Energy Barriers (Energies in eV)

\begin{tabular}{|l|c|c|c|c|}
\hline Authors & Method & Zr-Zr & Zr-Y & Y-Y \\
\hline $\begin{array}{l}\text { Krishnamurthy, } \\
\text { Yoon, Srolovitz } \\
\text { and Car }\end{array}$ & $\begin{array}{c}\text { Car-Parinello } \\
\text { MD }\end{array}$ & 0.58 & 1.29 & 1.86 \\
\hline $\begin{array}{l}\text { Krishnamurthy, } \\
\text { Srolovitz, } \\
\text { Kudin and Car }\end{array}$ & $\begin{array}{c}\text { Car-Parinello } \\
\text { MD }\end{array}$ & 0.473 & 1.314 & 2.017 \\
\hline \multicolumn{1}{|c|}{ This work } & DFT & 0.706 & 1.214 & 1.941 \\
\hline
\end{tabular}


kMC diffusivities as a function of Yttria concentration are shown below. The absolute values of the diffusivity are in general agreement with experiment and with other simulations.

Notably, the trend is consistent with experiment, and the maxima are in approximately the right places, and the location of the maximum increases with increasing $Y$ concentration, as observed experimentally.

This trend has been postulated to be the result of a competition between two effects.

(1) As Y concentration increases, the number of oxygen vacancies increases so as to maintain electrical neutrality. Increasing the available vacancies increases the diffusivity.(2) As $Y$ concentration increases, the number of relatively high-energy $\mathrm{Zr}-\mathrm{Y}$ and $\mathrm{Y}-\mathrm{Y}$ barriers increases, inhibiting diffusion.

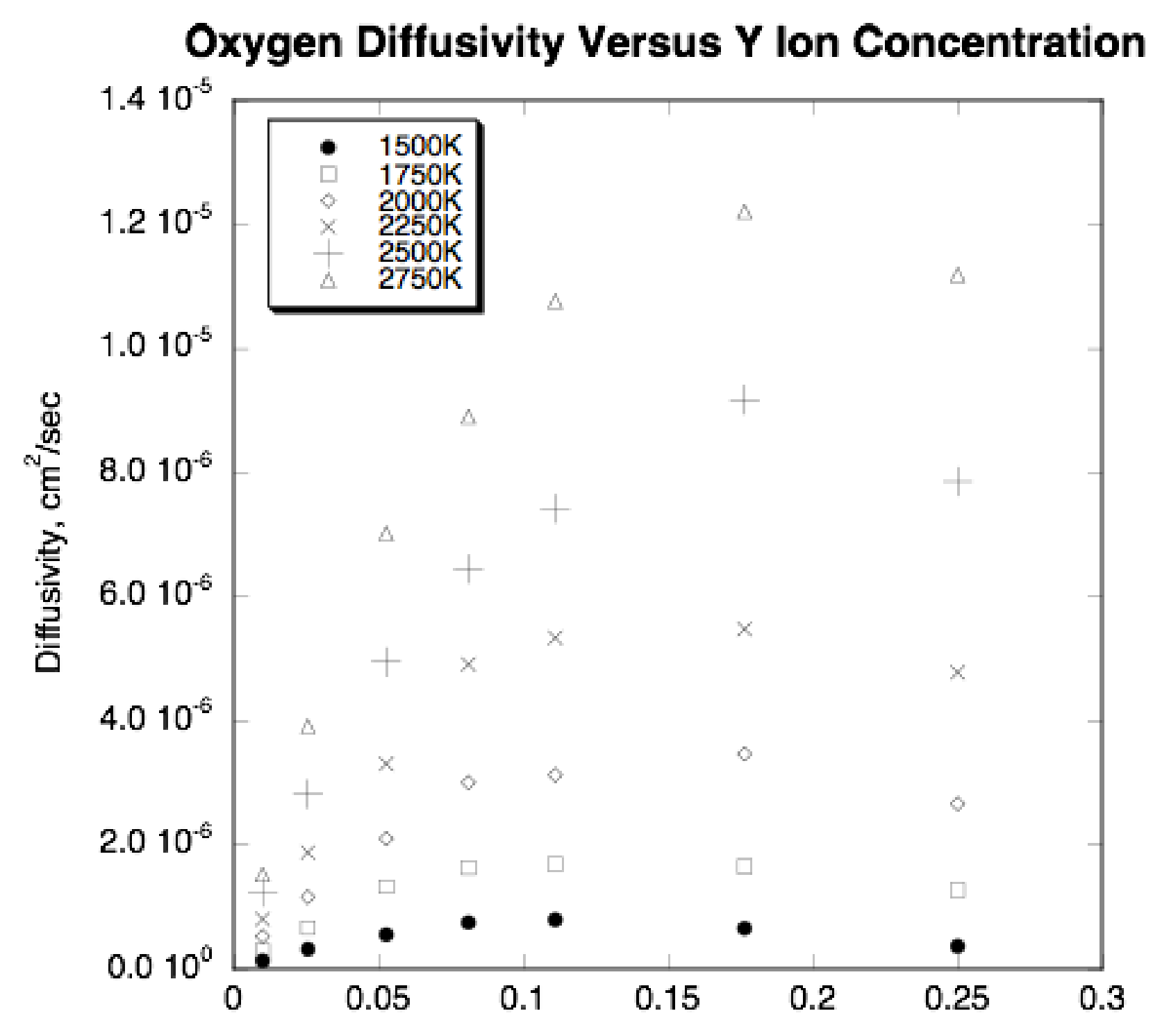


Temperature dependence of the oxygen diffusivity for a number of Yttria concentrations is shown below. Activation energies range from $0.25 \mathrm{eV}$ to $0.46 \mathrm{eV}$, in good agreement with experiment.

The activation energies increase with increasing Yttria concentration. This is consistent with the larger numbers of high energy $\mathrm{Zr}-\mathrm{Y}$ and $\mathrm{Y}-\mathrm{Y}$ barriers present at higher Yttria concentrations.

Temperature Dependence of Oxygen Diffusivity

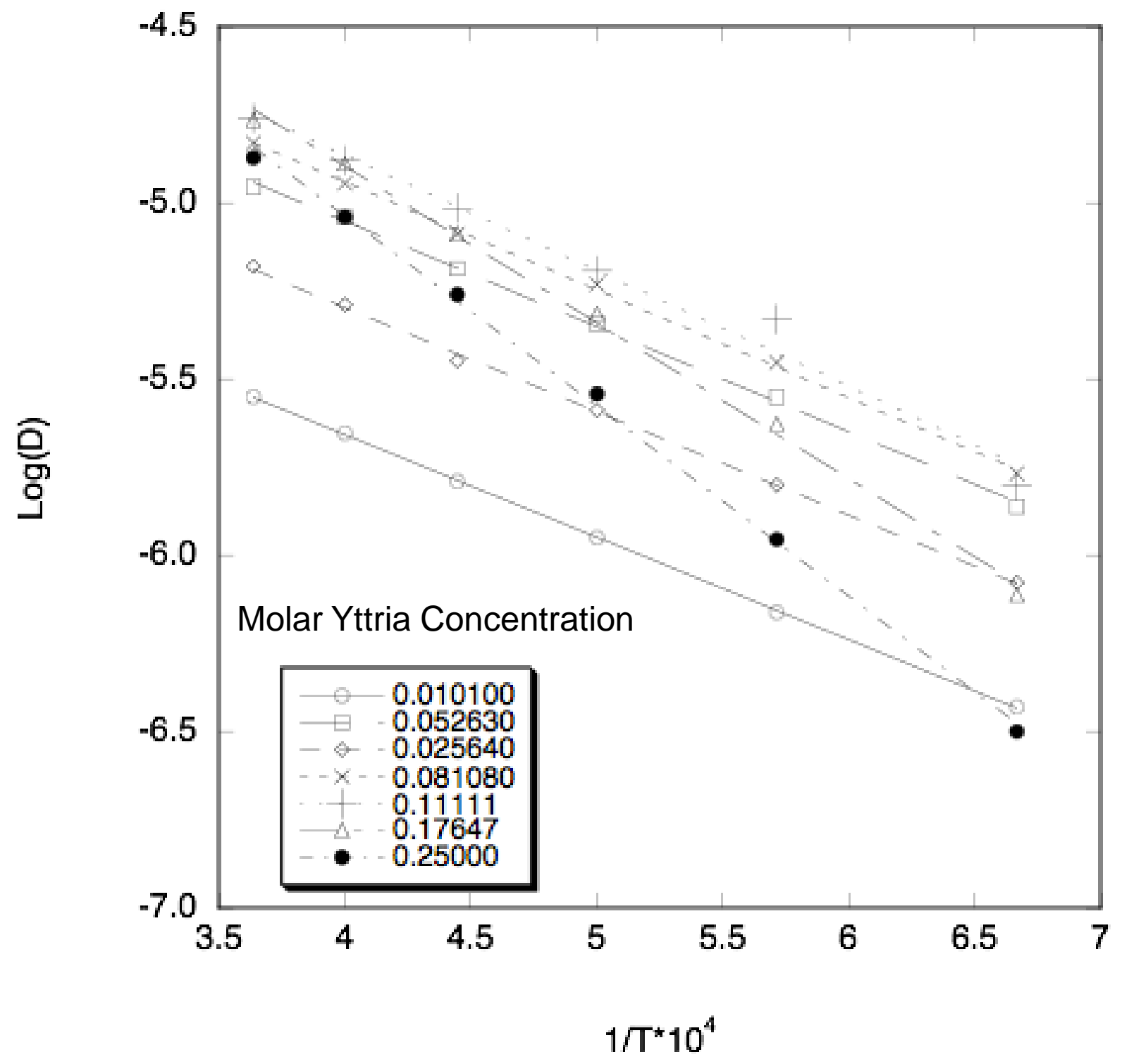


There is some indication that oxygen vacancies may preferentially occupy either yttrium or zirconium nearest neighbor sites. If so, this may affect the barrier energies. We have computed the change in barrier energy for two cases.

(1) A yttrium ion is a nearest neighbor to a hopping oxygen ion.

(2) An oxygen vacancy is a nearest neighbor to a yttrium barrier ion.

Barrier energies with preferential $\mathrm{O}$ vacancy- $\mathrm{Y}$ ion clustering (energies in $\mathrm{eV}$ )

\begin{tabular}{|c|c|c|c|}
\hline & $Z r-Z r$ & $Z r-Y$ & $Y-Y$ \\
\hline Original & 0.706 & 1.214 & 1.86 \\
\hline $\begin{array}{c}\text { Y adjacent to } \\
\text { hopping O }\end{array}$ & 0.691 & 1.140 & 1.941 \\
\hline $\begin{array}{c}\text { O vacancy } \\
\text { adjacent to } \mathrm{Y} \\
\text { barrier ion }\end{array}$ & & 1.0006 & 1.64 \\
\hline
\end{tabular}


There appears to be only a small effect on diffusivity at large $Y$ concentrations, but additional configurations are currently being investigated.

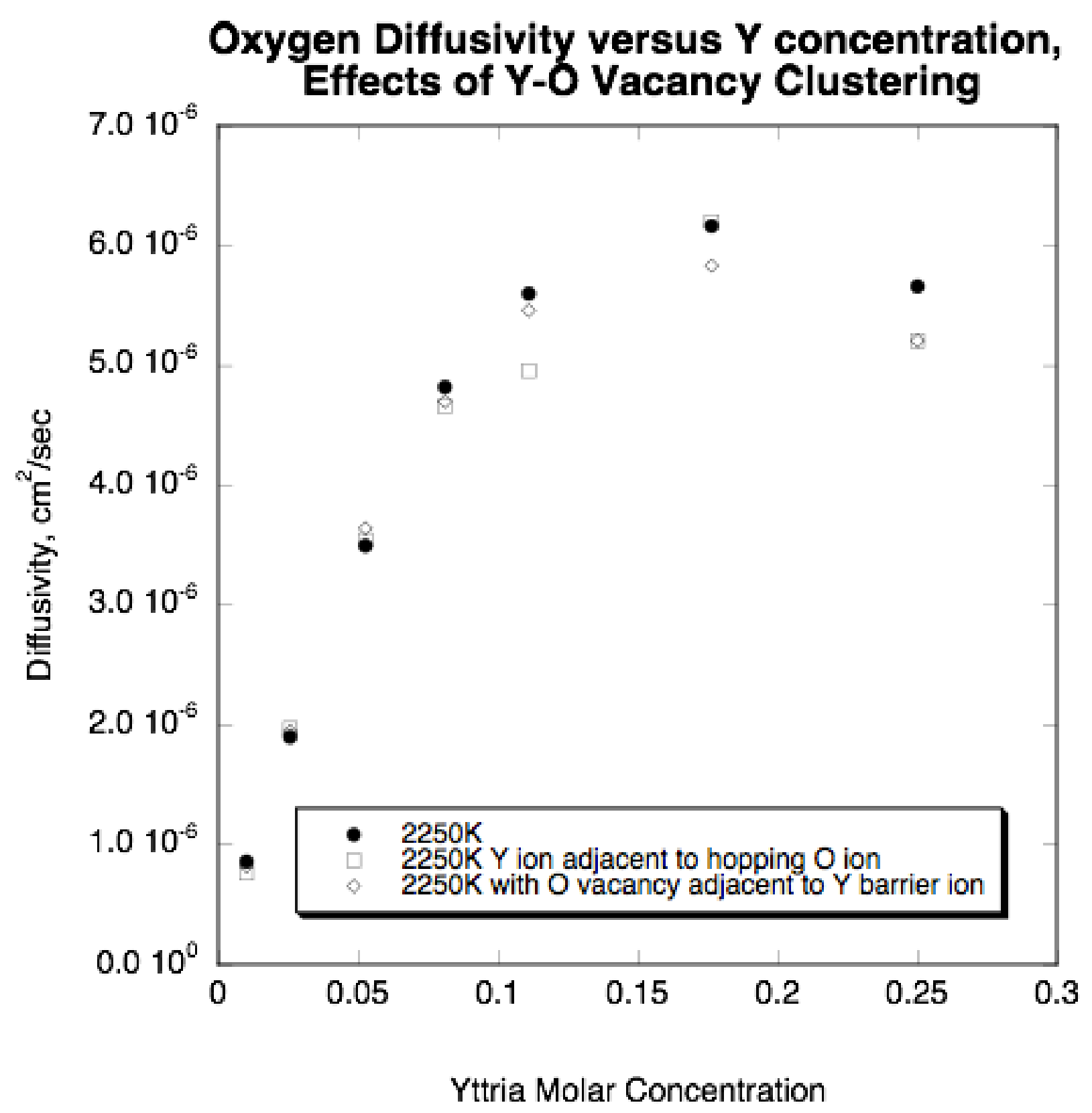




\section{Cation diffusion}

Cation diffusion is treated in a similar way, except that the diffusion path and energetics are different.

Initial ab initio calculations, along with MD results from the literature, suggest that nearest neighbor hops in the (110) direction are energetically favorable compared with longer (100) hops.

The barrier of oxygen ions consists of a single pair.
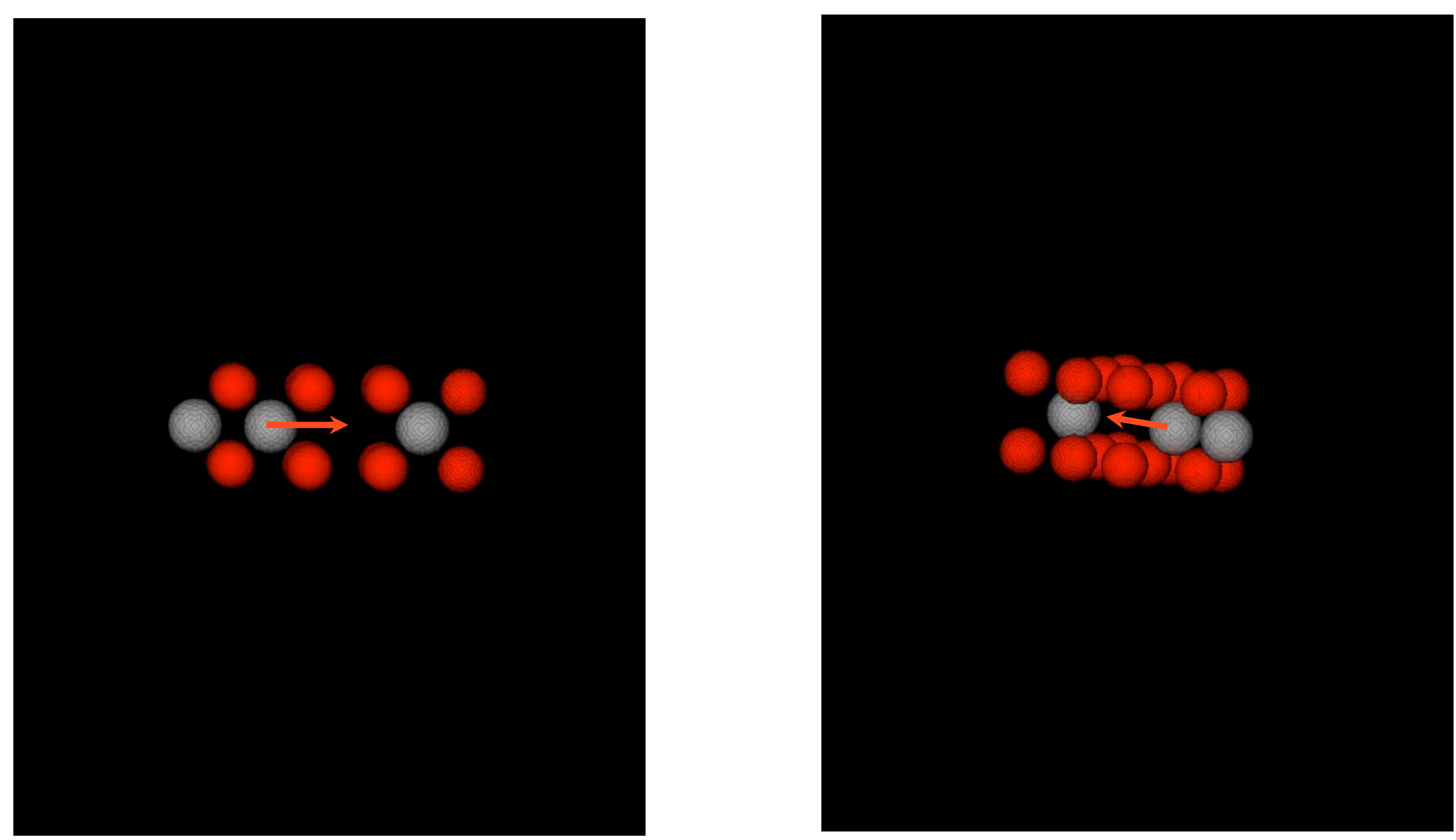

Cation hopping path. Oxygen ions are red, cations grey. 
$A b$ initio calculations to date yield barrier energies that are somewhat too large. A more detailed ab initio reaction path calculation may resolve the issue.

Experimental values of the hopping enthalpies, and the ordering of the $\mathrm{Zr}$ and $\mathrm{Y}$ enthalpies, are not consistent.

We have chosen barrier

energies of 3.7 and 3.62 for

$\mathrm{Y}$ and $\mathrm{Zr}$ migration, respectively. These values have been chosen to produce diffusivities within the range of experimental values at $2000 \mathrm{~K}$.

Note that the difference in barrier energies for $\mathrm{Zr}$ and $\mathrm{Y}$ hops is small.

\begin{tabular}{|l|l|l|}
\hline Author & $\begin{array}{c}\text { Temperature } \\
\text { Range, C }\end{array}$ & \multicolumn{1}{|c|}{ Value, eV } \\
\hline Solmon & $1300-1700$ & $4.8-4.95$ \\
\hline Gomez-Garcia & above 1500 & $5.5-6.0$ \\
\hline Chien and Heuer & $1100-1300$ & 5.3 \\
\hline Dimos and Kohlstedt & $1400-1600$ & 5.85 \\
\hline Kilo & $1125-1460$ & $4.4-4.8$ \\
\hline Mackrodt & & $\begin{array}{l}2-7 e V(\text { migration } \\
\text { energies })\end{array}$ \\
\hline This work & & $3.7(Y), 3.62$ (Zr) \\
\hline
\end{tabular}

H. Solmon, C. Monty, M. Filial, G. Petot-Ervas and C. Petot, Solid State Phenom. 41, 103 (1995).

D. Gomez-Garcia, J. Martinex-Fernandez, A. Dominguez-Rodriguez and J. Castaing, J. Am. Ceram. Soc. 80, 1668-1672 (1997).

F. R. Chien and A. H. Heuer, Philos. Mag. A 73, 681-697 (1996).

D. Dimos and D. L. Kohlstedt, J. Am. Ceram. Soc. 70, 277 (1987).

W. C. Mackrodt and P. M. Woodrow, J. Am. Ceram. Soc. 68, 277 (1986).

M. Kilo, G. Borchardt, C. Lesage, O. Kaitsov, S. Weber and S. Scherer, J. Eur. Ceram. Soc., Faraday Trans. 5, 2069 (2000).

M. Kilo, M. A. Taylor, C. Argirusis, G. Borchardt, R. A. Jackson, O Schulz, M. Martin and M. Weller, Solid State lonics 175, 823-827 (2004). 
Diffusivity is expected to depend on cation vacancy concentration and on $\mathrm{Y}$ concentration.

Cation diffusivity is expected to increase with cation vacancy concentration, but the number of such vacancies is small.

Because the barrier energies for $\mathrm{Zr}$ and $\mathrm{Y}$ hopping are not very different, the direct effect of increasing the $Y$ concentration is expected to be small.

Increasing the $Y$ concentration increases the oxygen vacancy concentration, which increases the probability that a vacancy will exist in the barrier oxygen pair. Ab initio calculations suggest that such vacancies will reduce the barrier energy, and increase the diffusivity.

Results from experiment and simulation are not conclusive, but only a weak concentration dependence is typically observed. 
The vacancy concentration on the cation sublattice is much smaller than on the oxygen sublattice. We consider cation vacancy concentrations of one percent or smaller.

Diffusivity versus temperature for our results and results from the literature are shown at right. The slope of the line from our kMC calculations is less than that of the experimental results shown, although it differs from the results of Chien et al. by less than ten percent. Other kMC simulation results (not shown) using larger barrier energies show a slope more consistent with other experimental results the reasonable agreement between simulation and experiment of the slopes of the $\ln (D)$ versus $1 / T$ suggests that the KMC simulations capture the fundamentals of the diffusion process.

Temperature Dependence of Cation Diffusivity

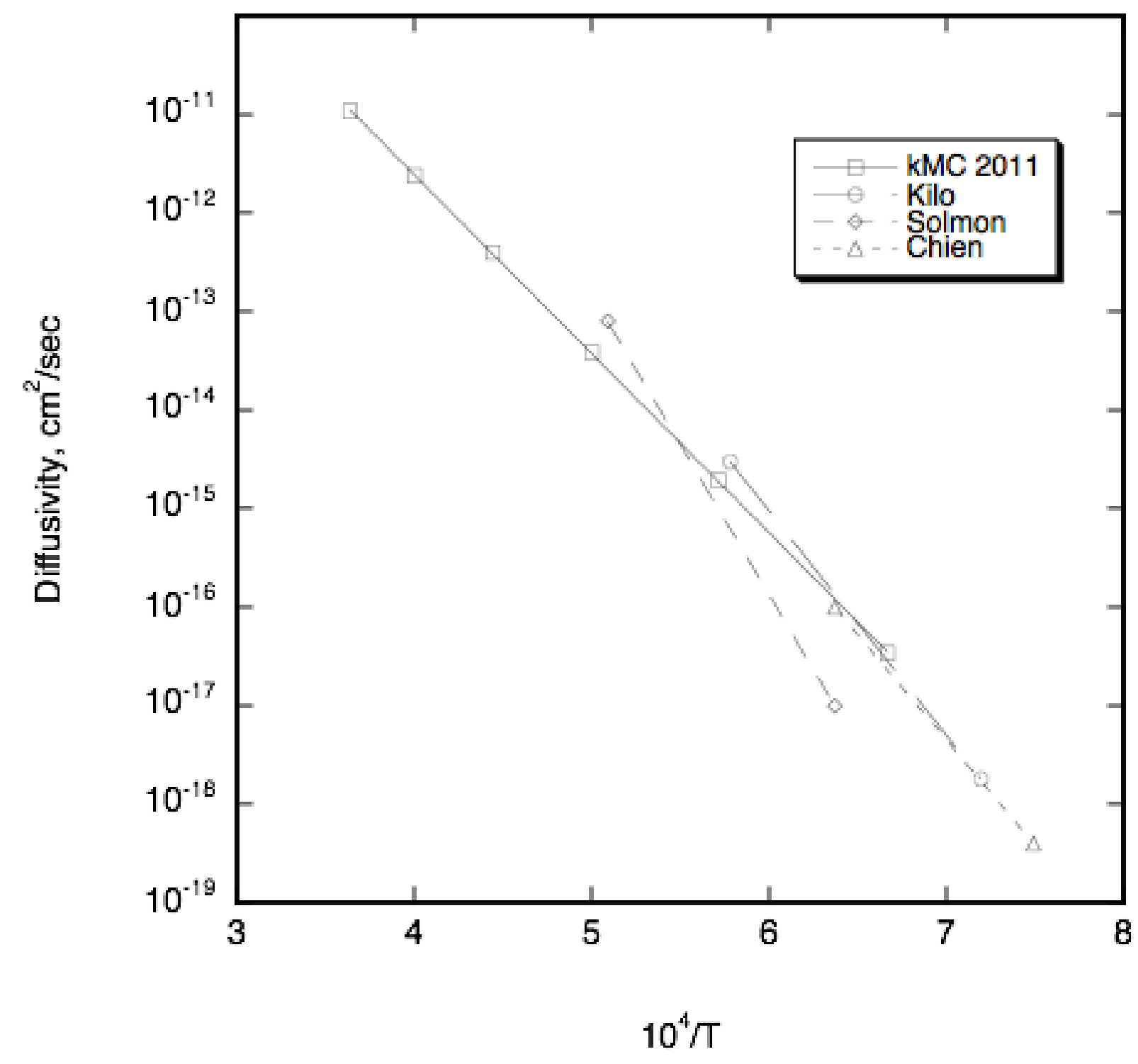




\section{Conclusions and Outlook}

kMC is capable of producing accurate simulations of "infrequent-event" phenomena, and provides insight into dynamical behavior without detailed atomic trajectories.

Oxygen diffusivities from $\mathrm{kMC}$ simulations using ab initio barrier energies are in reasonable agreement with experiment and other theoretical work.

There are small differences in the barrier energies depending on the cation species adjacent to the hopping oxygen ion or the barrier cations. Therefore it may be problematic to assume that oxygen vacancies and dopant cations are randomly distributed within the model lattice. It may be possible to generate more realistic distributions using a Metropolis Monte Carlo species exchange algorithm.

$A b$ initio barrier cation hopping energies for the assumed barrier complex are too large. Reaction path calculations may provide insight.

Using barrier energies derived from experiment produces cation diffusivities and temperature dependences that are in reasonable agreement with experiment.

We are currently extending our work to other dopants, and other TBC and EBC candidate materials.

Enlightening discussions with Beth Opila, Dongming Zhu, Nate Jacobson and Jim Smialek are gratefully acknowledged. 


\section{Possible additions to conclusion slide}

KMC simulations can produce realistic diffusivities and temperature and concentration dependences. 


\section{kMC, briefly}

All possible events of interest accessible to the system are identified, and the energetics computed.For each event, a probability, and the associated rate, are computed.All events and probabilities are organized into a catalog. Iteratively: An event is chosen at random from the catalog and executed. The simulation clock is advanced stochastically, consistent with the rates.

The event catalog is updated to reflect the new accessible events and their probabilities. 


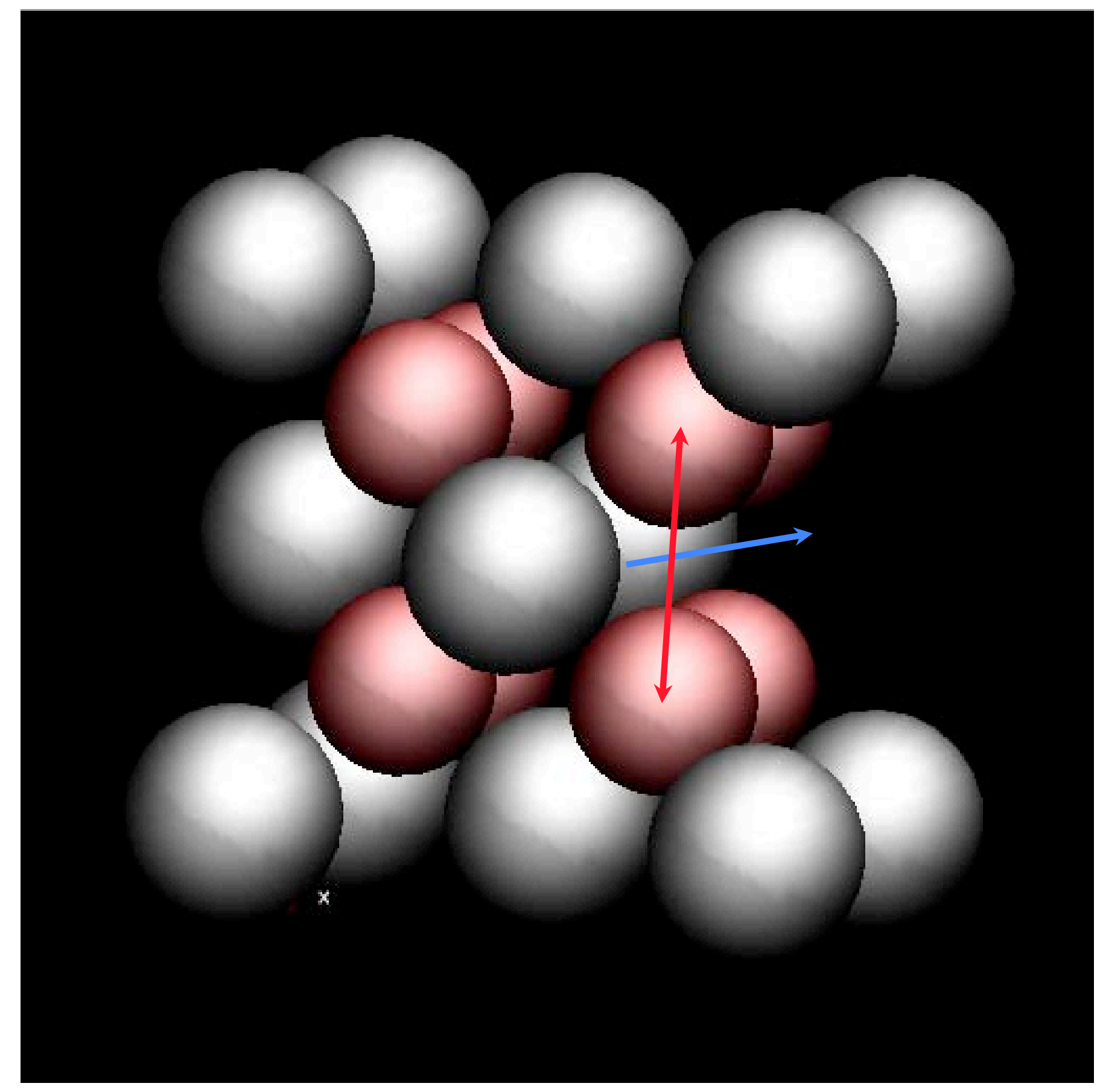




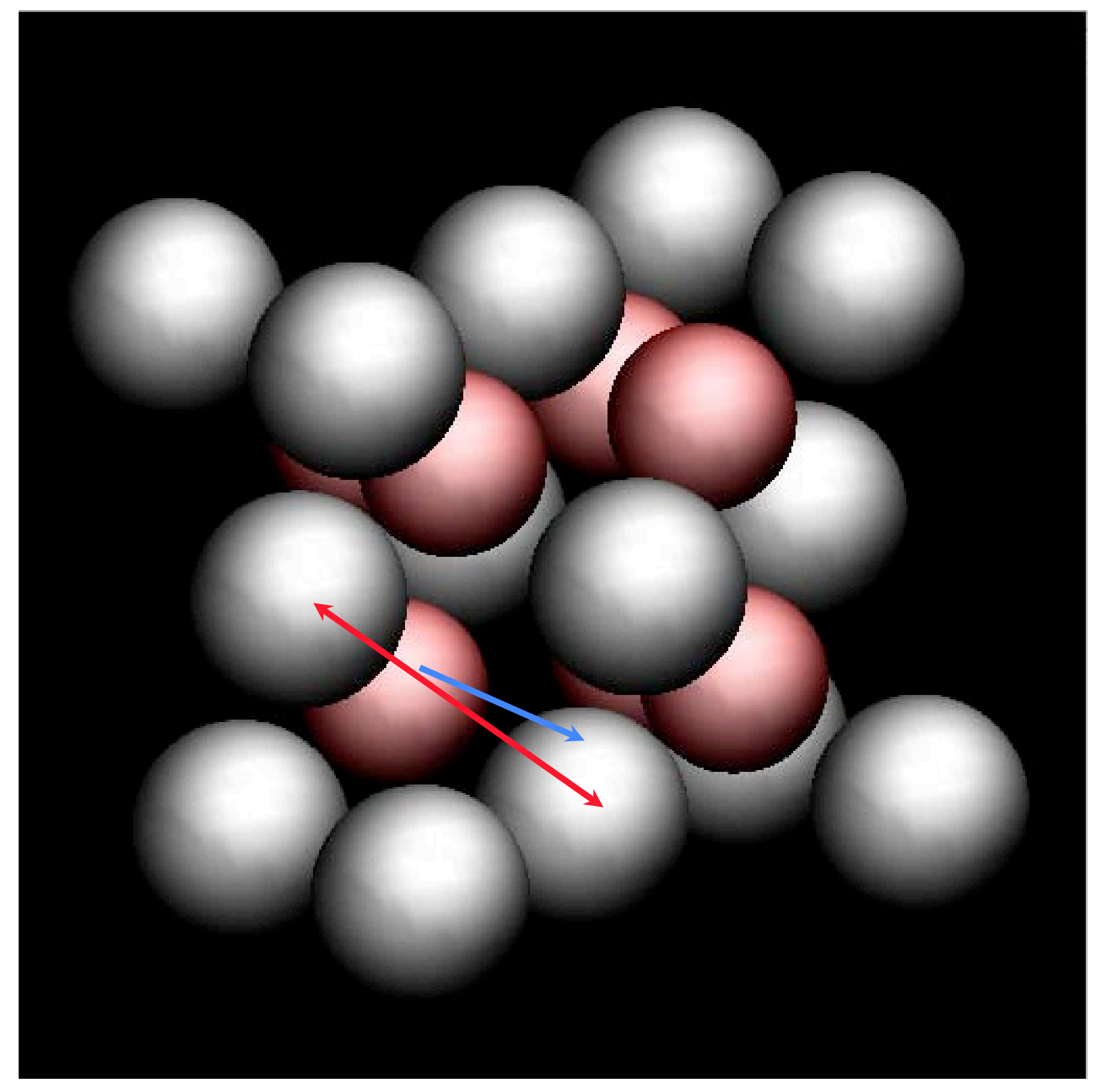

